\title{
Soft excesses of hard X-ray selected Seyfert 1 galaxies studied with the ROSAT-PSPC
}

\author{
L. Piro ${ }^{1}$, G. Matt ${ }^{1,2}$, and R. Ricci ${ }^{1, \star}$ \\ 1 Istituto di Astrofisica Spaziale - C.N.R., Via E. Fermi 21, I-00044 Frascati(RM), Italy \\ 2 Dipartimento di Fisica, Università degli Studi "Roma Tre", Via della Vasca Navale 84, I-00146 Roma, Italy
}

Received January 8; accepted April 17, 1997

\begin{abstract}
The nature of the soft X-ray excess in Seyfert 1 galaxies is still an open issue. In order to derive some general properties we analysed the ROSAT PSPC archive observations of the Seyfert galaxies in the Nandra \& Pounds (1994) Ginga sample. The use of this sample ensures a reliable estimate (under the assumption of lack of intrinsic spectral variability) of the slope of the underlying power law, which instead cannot be well measured using ROSAT data alone.

We tested three different models for the soft excess: emission from accretion disk (described by a black body), absorption from ionized gas ("warm absorber", described by an absorption edge), and reflection from mildly ionized material (described by a step function). The main result is that no model gives a satisfactory fit for all sources. Furthermore for two sources none of these models provides an acceptable fit. In these cases a physically meaningful combination of two components (e.g. warm absorber plus black body) gives a good fit. We conclude that several different effects concur to the soft excess phenomenon in Seyfert galaxies.
\end{abstract}

Key words: galaxies: Seyfert; X-rays: galaxies

\section{Introduction}

Soft X-ray excesses, i.e. excess emission in the soft $(\lesssim 1 \mathrm{keV}) \mathrm{X}$-ray band with respect to the extrapolation of the spectrum at higher energies, are fairly common in radio-quiet Active Galactic Nuclei (AGN). Turner \& Pounds (1989) found that about $50 \%$ of the sources in their EXOSAT sample exhibits a significant low energy spectral steepening. An analysis of ROSAT All Sky Survey sources by Walter \& Fink (1993) has shown that the

\footnotetext{
Send offprint requests to: Luigi Piro: piro@alpha1.ias.fra.cnr.it * Present Address: SAX/SOC Nuova Telespazio, Via Corcolle 19, I-00131 Roma, Italy.
}

ROSAT PSPC $(0.1-2.4 \mathrm{keV})$ spectral index is significantly steeper than the typical one at higher energies for about $90 \%$ of the objects.

The spectral steepening in the soft $\mathrm{X}$-ray band has been originally attributed to a real emission component, i.e. thermal emission from an accretion disk (i.e. Arnaud et al. 1985). Later on, the relevance of absorption features due to ionized matter (i.e. the "warm absorber", Halpern 1984) in modifying the spectral shape in soft $\mathrm{X}$-rays has also been recognized in some sources (e.g. Nandra \& Pounds 1992). More recently an alternative explanation based on reflection by ionized media has been successfully applied to a few AGN (Piro et al. 1997; Życki et al. 1994; Ross \& Fabian 1993).

On the other hand, the systematic analysis of Walter \& Fink indicates that the shape of this component is similar in all the sources of their sample, suggesting a common origin. Unfortunately, while the ROSAT-PSPC has been of valuable importance in establishing the presence of soft excesses, its limited bandwidth and energy resolution do not permit to study in detail their shape and strength. To this aim, an independent knowledge of the underlying hard X-ray power law would be extremely important, this component being not easily measurable by ROSAT. For a few sources, simultaneous ROSAT-Ginga observations were available. Most of them have been obtained during the ROSAT All Sky Survey (RASS); owing to the limited sensitivity of the RASS data, the conclusions proposed by two different groups (Walter et al. 1994; Pounds et al. 1994) on the origin of the soft X-ray excess were somewhat different.

We have therefore performed a systematic study of the spectra of the pointed observations contained in the ROSAT archive of a sample of hard X-ray selected sources, whose $2-10 \mathrm{keV}$ spectrum is well known from not simultaneous Ginga observations (Nandra \& Pounds 1994). In our analysis we have assumed that the slope of the intrinsic power law remains constant, leaving its normalization as a free parameter to account for flux variations. In fact, convincing evidence for spectral variability 
of the intrinsic power law has been found so far only in two objects, namely NGC 4151 (Perola et al. 1986; Yaqoob \& Warwick 1991), which is not part of our sample, and NGC 4051 (Guainazzi et al. 1997). We will comment specifically on the latter source in Sect. 4; we anticipate here that for this object the results are not significantly affected by the choice of the intrinsic slope.

The paper is organized as follows: the sample selection, ROSAT observations and data reduction are discussed in Sect. 2. The results are described in Sect. 3, while Sect. 4 is devoted to individual sources and Sect. 5 to the discussion and the conclusions.

\section{Sample selection and data analysis}

\subsection{The Ginga sample}

We first selected a sample consisting of the sources among the Nandra \& Pounds (1994) Ginga sample classified as pure Seyfert 1 galaxies. Seyfert 1.5, 2 and Narrow Emission Line Galaxies are excluded: generally these sources are highly absorbed and are therefore very faint in the ROSAT energy range. We have also been forced to exclude the Seyfert 1 3C 382 because no ROSAT PSPC observation is available. This led to the selection of 17 low redshift $(z<0.06)$ sources spanning a wide range of luminosities (from a few $\times 10^{41}$ up to almost $10^{45} \mathrm{erg} \mathrm{s}^{-1}$ ). The main source characteristics, as well as the date of Ginga observation (or the dates of the first and last observations if more than one) are listed in Table 1. From the Nandra $\&$ Pounds analysis we have used the slope of the intrinsic power law derived by including in the model a reflection component and, when relevant, a warm absorber (Table 10 of their paper and last column of our Table 2).

\subsection{ROSAT data analysis and sample selection}

Of all these 17 sources we have then analized the PSPC (Pfeffermann et al. 1987) observations in the ROSAT (Trümper 1983) public archive. In case of repeated pointings we have selected the longest observation, because we are primarily interested in the spectral properties. The log book of the ROSAT observations can also be found in Table 1.

Data of each observation were analyzed using the EXSAS package (Zimmermann et al. 1994). After examination of the image to exclude serendipitous sources that may contaminate source and background extraction regions, source spectra were extracted from a circular region of 3 arcmin radius. The background was computed in an annulus between 3.5 and 7 arcmin. The date of observation, exposure times, total source counts and count rates are listed in Table 1. For each source we produced a 256 channel pulse-invariant (PI) spectrum. We ignored PI channels $1-11(E<0.1 \mathrm{keV})$ and above 235 $(E>2.36 \mathrm{keV})$ and rebinned the remaining channels in order to have a signal to noise ratio in each bin of at least
7. We have added systematic errors of $2 \%$ to account for residual calibration uncertainties (ROSAT NEWS No. 10, Aug. 1992). The spectral analysis was performed using the PSPC response matrix released on Mar. 92 for the observations performed before 21 Nov. 1991, and the matrix released on Jan. 1993 for observations performed after that date.

In order to have adequate statistics for spectral analysis, particularly below $1 \mathrm{keV}$, we have further selected objects with ROSAT PSPC total counts greater than 2000 cts and absorption column density lower than $10^{21} \mathrm{~cm}^{-2}$. Two objects do not satisfy the first requirement (Table 1): NGC 4593 and 3C 111. As for the second selection criterion, we present in Table 2 the results of the simple power law fits to the 15 remaining objects. One object, IC 4329A, has an absorbing column in excess of $10^{21} \mathrm{~cm}^{-2}$ : the final sample is therefore composed of 14 objects, with total counts comprised between 12.000 and 65.000 cts.

Hereinafter all the errors refer to a $90 \%$ confidence level for one interesting parameter.

\section{Results}

\subsection{Fit with simple power law and comparison with Ginga spectral index}

In Table 2 we present the results of the fit with a simple power law with $\Gamma$ and $N_{\mathrm{H}}$ as free parameters. 7 sources have $\chi_{\nu}^{2}>1.25$. From the $\chi^{2}$ statistics (with $\nu \simeq 110-$ 200) we would expect to have only 2 with values of $\chi^{2}$ greater than 1.25 , were the model statistically acceptable. We therefore conclude that the simple power law model is not an adequate representation of the spectral shape of the sample in the ROSAT bandpass.

Furthermore, as can be seen in Fig. 1 and Table 2, the power law indices are usually greater than those of Ginga. The average values are respectively $\Gamma_{\text {ROSAT }}=2.38 \pm 0.01$, $\Gamma_{\text {Ginga }}=1.97 \pm 0.01$. Only for one object, $3 \mathrm{C} 390.3$, the spectrum in the ROSAT range is well described by a simple power law with a photon index consistent with that observed by Ginga. This result agrees with the Walter \& Fink (1993) finding of about $90 \%$ of the sources having a soft excess.

The column densities agree in general very well with the Galactic ones, after taking into account the typical error of about $110^{20} \mathrm{~cm}^{-2}$ on the determination on $N_{\mathrm{HGal}}$. In Fig. 2 we present the distribution of $N_{\mathrm{H}}-N_{\mathrm{HGal}}$. The average value is $0.34 \pm 0.1510^{20} \mathrm{~cm}^{-2}$ ( $1 \sigma$ error $)$ with a standard deviation of $0.6910^{20} \mathrm{~cm}^{-2}$. Only 3C 390.3 shows a marginal evidence of an intrinsic absorption of the order of $210^{20} \mathrm{~cm}^{-2}$.

\subsection{Modeling the soft $X$-ray excess}

Once established the common presence of a soft excess, we attempted, for the 13 out of 14 sources which have a ROSAT power law index different from the Ginga index 
Table 1. Log of Ginga and ROSAT observations

\begin{tabular}{|c|c|c|c|c|c|c|c|}
\hline Obj. & $z$ & $N_{\text {Hgal }}^{1}$ & \multicolumn{2}{|l|}{ Ginga } & \multicolumn{3}{|l|}{ ROSAT } \\
\hline Mkn 335 & 0.026 & 3.96 & $12 / 87-06 / 91$ & 29-06-91 & 24586 & 65989 & $2.68 \pm 0.01$ \\
\hline Fairall-9 & 0.046 & 2.82 & $11 / 90$ & $10-05-91$ & 8173 & 31834 & $3.89 \pm 0.02$ \\
\hline NGC 3783 & 0.01 & 9.41 & $01 / 90$ & 08-07-93 & 23344 & 38051 & $1.63 \pm 0.01$ \\
\hline NGC 4051 & 0.002 & 1.37 & $06 / 87-11 / 90$ & $16-11-91$ & 28727 & 46509 & $1.62 \pm 0.01$ \\
\hline NGC 4593 & 0.009 & 1.97 & $06 / 87-12 / 87$ & $14-07-92$ & 1261 & 1849 & $1.47 \pm 0.03$ \\
\hline MCG 6-30-15 & 0.008 & 4.06 & $09 / 87-02 / 90$ & 29-01-92 & 8513 & 17017 & $2.00 \pm 0.02$ \\
\hline Mkn 841 & 0.036 & 2.24 & $07 / 90-01 / 91$ & 20-01-92 & 17185 & 36656 & $2.13 \pm 0.01$ \\
\hline 3C 390.3 & 0.056 & 4.12 & $11 / 88$ & $17-03-91$ & 11516 & 12184 & $1.06 \pm 0.01$ \\
\hline NGC 7469 & 0.017 & 4.82 & $06 / 88$ & 28-05-92 & 18575 & 32933 & $1.77 \pm 0.01$ \\
\hline Akn 120 & 0.033 & 11.3 & $09 / 88$ & 18-09-91 & 24493 & 36127 & $1.47 \pm 0.01$ \\
\hline NGC 5548 & 0.017 & 1.93 & $06 / 88-07 / 90$ & $16-07-90$ & 18861 & 34440 & $1.83 \pm 0.01$ \\
\hline Mkn 509 & 0.036 & 3.99 & $10 / 88-10 / 90$ & $29-10-92$ & 2294 & 12534 & $5.46 \pm 0.05$ \\
\hline NGC 3516 & 0.009 & 3.55 & $10 / 89-10 / 89$ & 03-10-92 & 13081 & 61206 & $4.68 \pm 0.02$ \\
\hline IC $4329 \mathrm{~A}$ & 0.014 & 4.55 & $07 / 89$ & 04-01-93 & 8230 & 23307 & $2.83 \pm 0.02$ \\
\hline MCG $2-58-22$ & 0.048 & 3.51 & $06 / 89-11 / 89$ & $21-05-93$ & 18767 & 17322 & $0.92 \pm 0.01$ \\
\hline NGC 7213 & 0.006 & 1.94 & $06 / 90-19 / 90$ & $10-05-91$ & 6688 & 36316 & $5.43 \pm 0.03$ \\
\hline $3 \mathrm{C} 111$ & 0.048 & 32.61 & $02 / 89$ & $13-02-93$ & 2322 & 1001 & $0.43 \pm 0.01$ \\
\hline
\end{tabular}

Note: (1) Galactic column density in units of $10^{20} \mathrm{~cm}^{-2} ;(2)$ in s.; (3) in counts/s.

Table 2. Fit with a simple power law

\begin{tabular}{llllll}
\hline Obj. & $C_{\mathrm{pl}}^{1}$ & $N_{\mathrm{H}}^{2}$ & $\Gamma_{\text {ROSAT }}$ & $\chi_{\nu}^{2} / \nu$ & $\Gamma_{\text {GINGA }}^{3}$ \\
Mkn 335 & $6.70 \pm 0.10$ & $3.88 \pm 0.13$ & $3.01 \pm 0.05$ & $1.52 / 176$ & $2.39 \pm 0.15$ \\
Fairall-9 & $11.4 \pm 0.15$ & $2.97 \pm 0.13$ & $2.46 \pm 0.05$ & $1.04 / 186$ & $1.94 \pm 0.09$ \\
NGC 3783 & $9.14 \pm 0.12$ & $10.0 \pm 0.29$ & $2.79 \pm 0.07$ & $17.5 / 111$ & $2.11 \pm 0.07$ \\
NGC 4051 & $2.24 \pm 0.04$ & $1.79 \pm 0.10$ & $2.84 \pm 0.04$ & $1.66 / 174$ & $1.84 \pm 0.09$ \\
MCG 6-30-15 & $8.73 \pm 0.15$ & $4.83 \pm 0.24$ & $2.23 \pm 0.07$ & $2.46 / 189$ & $2.12 \pm 0.04$ \\
Mkn 841 & $5.28 \pm 0.08$ & $2.28 \pm 0.11$ & $2.42 \pm 0.04$ & $1.25 / 187$ & $2.30 \pm 0.02$ \\
3C 390.3 & $5.63 \pm 0.13$ & $6.33 \pm 0.35$ & $1.89 \pm 0.07$ & $1.15 / 177$ & $1.86 \pm 0.03$ \\
NGC 7469 & $8.63 \pm 0.11$ & $5.82 \pm 0.18$ & $2.29 \pm 0.05$ & $1.03 / 199$ & $1.97 \pm 0.05$ \\
Akn 120 & $9.13 \pm 0.14$ & $10.2 \pm 0.31$ & $2.53 \pm 0.05$ & $0.93 / 199$ & $1.93 \pm 0.11$ \\
NGC 5548 & $4.56 \pm 0.06$ & $1.33 \pm 0.09$ & $1.94 \pm 0.04$ & $1.84 / 196$ & $1.81 \pm 0.02$ \\
Mkn 509 & $17.7 \pm 0.38$ & $3.93 \pm 0.23$ & $2.63 \pm 0.08$ & $0.96 / 158$ & $1.86 \pm 0.02$ \\
NGC 3516 & $15.6 \pm 0.16$ & $2.78 \pm 0.10$ & $2.15 \pm 0.04$ & $3.28 / 208$ & $2.08 \pm 0.09$ \\
IC 4329A & $23.2 \pm 2.10$ & $23.5 \pm 3.2$ & $1.30 \pm 0.15$ & $0.92 / 197$ & $2.03 \pm 0.04$ \\
MCG 2-58-22 & $3.59 \pm 0.06$ & $3.51 \pm 0.18$ & $2.11 \pm 0.06$ & $0.96 / 183$ & $1.67 \pm 0.08$ \\
NGC 7213 & $15.8 \pm 0.20$ & $2.15 \pm 0.10$ & $2.14 \pm 0.04$ & $1.55 / 195$ & $1.96 \pm 0.08$ \\
\hline
\end{tabular}

Note: (1) Power law flux at $1 \mathrm{keV}$ in units of $10^{-3} \mathrm{ph} \mathrm{cm}^{-2} \mathrm{~s}^{-1} \mathrm{keV}^{-1}$ (2) Neutral column density in units of $10^{20} \mathrm{~cm}^{-2}(3)$ Best fit power law index with reflection and warm absorber included.

or an unacceptable simple power law model, to fit the data with a two components model, i.e. consisting of a hard power law (with the spectral index now fixed to the Ginga value) plus a further component describing the soft excess.

Three spectral models, representing the most common physical interpretation of the soft excess, have been investigated:
- A black body, representing soft X-ray intrinsic emission from a distinct region, possibly the high energy tail of the thermal accretion disc emission.

- An absorption edge, representing absorption of the primary emission by partially ionized matter, i.e. the socalled "warm absorber". To be physically meaningful, the energy of the edge must correspond to that of a highly ionized element with a significant abundance (oxygen is the best candidate, but also carbon would 


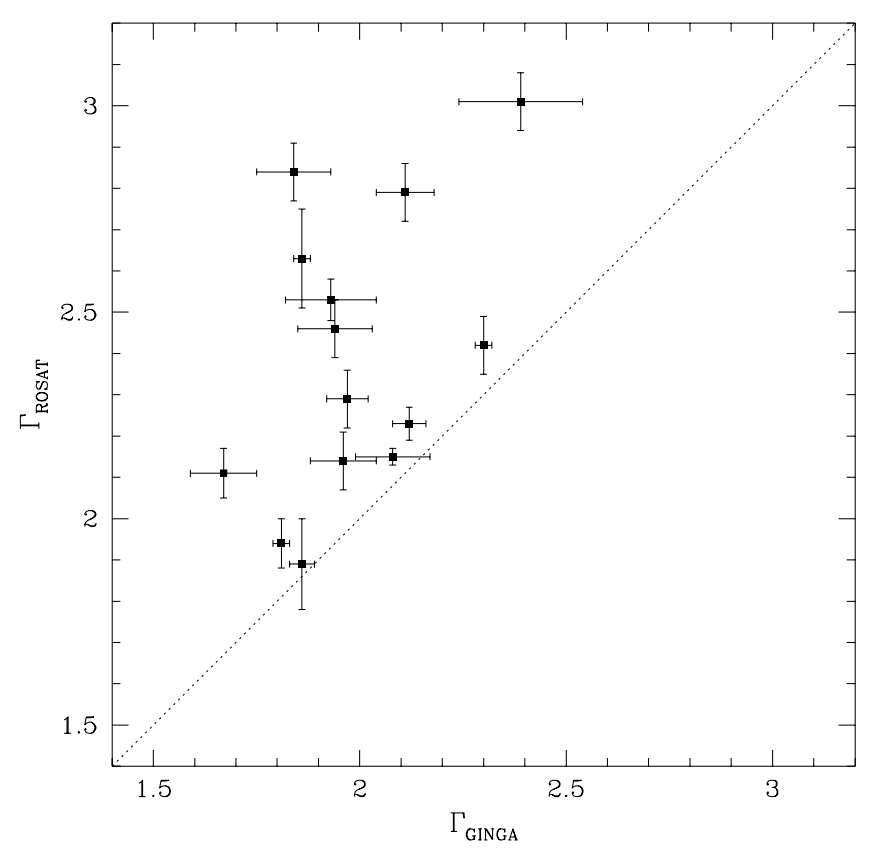

Fig. 1. Distribution of spectral index from ROSAT simple power law fits vs. Ginga. Note as the ROSAT slopes are sistematically greater than the Ginga slopes

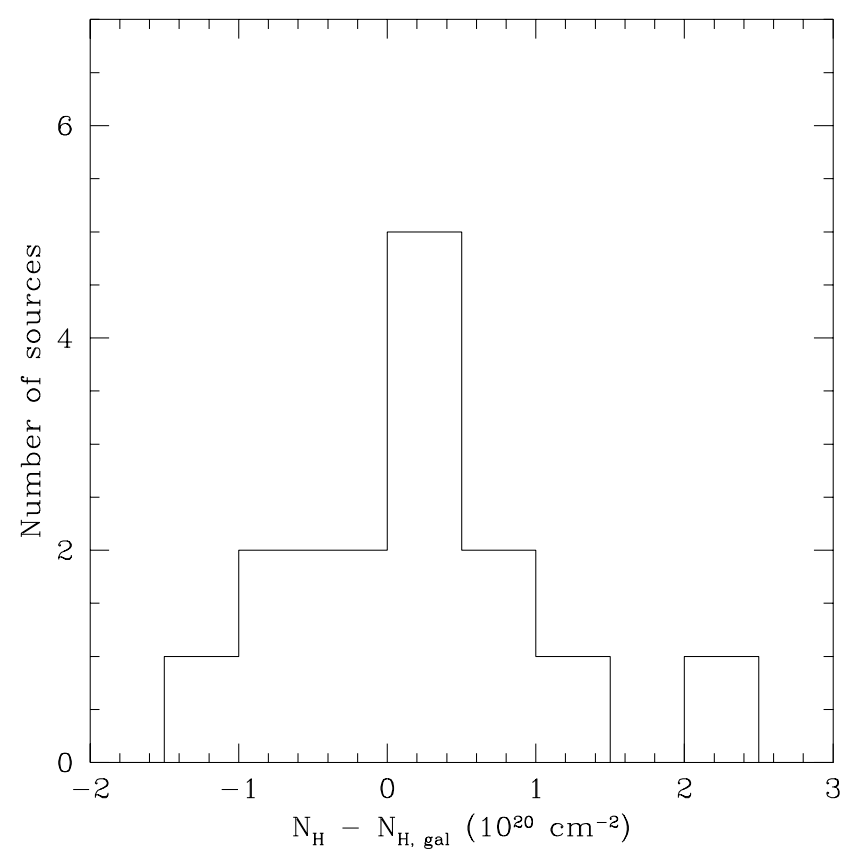

Fig. 2. Distribution of $N_{\mathrm{H}}-N_{\mathrm{HGal}}$ for the 14 objects in the sample be acceptable). It could be useful to recall that the edge energies are $0.39,0.49,0.74$ and $0.87 \mathrm{keV}$ for $\mathrm{C}$ V, C vi, O viI and O viII, respectively (Allen 1973).

- A reflection model, representing the case in which the primary emission is reflected by partially ionized matter (e.g. again the accretion disc). We parametrized this model in the simplest possible way, e.g. as a component identical with the intrinsic one (the hard power law) multiplied by a step function $\Theta\left[-\left(E-E_{0}\right)\right], E_{0}$ representing a photoabsorption edge energy (generally carbon or oxygen). More refined models are available in XSPEC but the added information they could potentially provide cannot be extracted due to the limited spectral resolution of the ROSAT PSPC. Then, after having verified that the results were basically the same, we prefer to present the most straightforward results of the simple model. Similarly to the absorption edge model, also in the reflection model we will consider the fit acceptable only when $E_{0}$ corresponds to a highly ionized ion of carbon or oxygen. Finally, it should be recalled that recently a detailed reflection model, based on Ross \& Fabian (1993) and Matt et al. (1993) models has been applied to the high state of the Seyfert 1 galaxy E1615+061 (Piro et al. 1997) and to a sample of quasars, providing acceptable fits only for the sources with lower optical luminosities (Fiore et al. 1997, where the model is described). This model is not very flexible, and above all it is valid only for highly ionized matter, while for the sources analyzed here, which have $\mathrm{X}$-ray luminosity on average much smaller than those of the Fiore et al. sample, it is likely that the disc is at most mildly ionized (Piro et al. 1997). For all these reasons, we prefer to ignore this model here.

The results of the analysis are presented in Table 3, Table 4 and Table 5 for the black body, edge and reflection models, respectively.

The parameterization adopted for the three physical models can appear rather simplistic (especially for the warm absorber and reflection models, for which line emission could also be expected), but it is possibly adequate for the energy resolution of the PSPC. In any case, we have checked it by adding a narrow gaussian line to the edge or reflection models for all sources for which these models do not give acceptable results. As described in Sect. 4.5, the addition of the line does not in general make the fit acceptable.

In Table 6 we summarize the applicability of each model to the sample of spectra; a cross means that the fit is statistically acceptable (we define as statistically "acceptable" any fit for which $\left.\chi_{\mathrm{r}}^{2}<1.25\right)$. 3C 390.3 is not included in the Table because no model gives a fit significantly better than the simple power law. It is worth noticing that for two sources, NGC 3783 and Fairall 9, all models fail to successfully fit the data. We will come back to this point in the following. A first, very important 
Table 3. Fit with black body

\begin{tabular}{llllll}
\hline Obj. & $C_{\mathrm{pl}}^{1}$ & $N_{\mathrm{H}}^{2}$ & $C_{\mathrm{bb}}^{3}$ & $k T^{4}$ & $\chi_{\nu}^{2}$ \\
Mkn 335 & $6.06 \pm 0.15$ & $3.55 \pm 0.24$ & $3.51 \pm 0.68$ & $74.6 \pm 3.9$ & 1.13 \\
Fairall-9 & $9.58 \pm 0.27$ & $2.11 \pm 0.15$ & $1.81 \pm 0.25$ & $105.0 \pm 7.8$ & 1.26 \\
NGC 3783 & $8.73 \pm 0.19$ & $15.90 \pm 0.70$ & $33.4 \pm$ & $63.4 \pm 1.9$ & 5.84 \\
NGC 4051 & $1.67 \pm 0.08$ & $0.83 \pm 0.13$ & $1.01 \pm 0.10$ & $92.7 \pm 3.8$ & 0.97 \\
MCG 6-30-15 & $9.18 \pm 0.29$ & $10.03 \pm 0.98$ & $25 \pm 12$ & $55.3 \pm 3.6$ & 1.38 \\
Mkn 841 & $5.25 \pm 0.10$ & $2.75 \pm 0.26$ & $1.25 \pm 0.52$ & $58.8 \pm 7.3$ & 1.02 \\
NGC 7469 & $7.67 \pm 0.19$ & $5.21 \pm 0.24$ & $0.87 \pm 0.22$ & $107 \pm 11$ & 0.91 \\
Akn 120 & $6.69 \pm 0.25$ & $8.05 \pm 0.26$ & $1.08 \pm 0.11$ & $140 \pm 10$ & 0.89 \\
NGC 5548 & $4.51 \pm 0.08$ & $1.92 \pm 0.21$ & $0.76 \pm 0.24$ & $59.3 \pm 7.3$ & 1.29 \\
Mkn 509 & $13.79 \pm 0.63$ & $2.67 \pm 0.29$ & $4.21 \pm 0.79$ & $103.2 \pm 8.7$ & 1.08 \\
NGC 3516 & $15.70 \pm 0.26$ & $4.79 \pm 0.16$ & $9.4 \pm 2.9$ & $60.9 \pm 3.3$ & 2.07 \\
MCG 2-58-22 & $3.03 \pm 0.12$ & $2.64 \pm 0.25$ & $0.36 \pm 0.08$ & $120 \pm 16$ & 1.01 \\
NGC 7213 & $15.9 \pm 0.3$ & $3.23 \pm 0.26$ & $5.8 \pm 2.3$ & $51.6 \pm 4.9$ & 1.08 \\
\hline
\end{tabular}

Note: (1) Power law flux at $1 \mathrm{keV}$ in units of $10^{-3} \mathrm{ph} \mathrm{cm}^{-2} \mathrm{~s}^{-1} \mathrm{keV}^{-1}$ (2) Neutral column density in units of $10^{20} \mathrm{~cm}^{-2}(3)$ Normalization of black body component $\left(C_{\mathrm{bb}}=L_{39} / D_{10}^{2}\right)$ in units of $10^{-3} \mathrm{erg} \mathrm{s}^{-1} / \mathrm{kpc}^{2}$ (4) Black body temperature (eV).

Table 4. Fit with absorption edge

\begin{tabular}{llllll}
\hline Obj. & $N_{\mathrm{H}}$ & $E^{2}$ & $\tau^{3}$ & $C_{\mathrm{pl}}^{4}$ & $\chi_{\nu}^{2}$ \\
Mkn 335 & $2.74 \pm 0.05$ & $0.93 \pm 0.02$ & $0.98 \pm 0.09$ & $8.57 \pm 0.19$ & 1.97 \\
Fairall-9 & $1.9 \pm 0.05$ & $1.15 \pm 0.05$ & $0.72 \pm 0.8$ & $12.8 \pm 0.3$ & 1.74 \\
NGC 3783 & $9.40 \pm 0.14$ & $0.84 \pm 0.01$ & $2.34 \pm 0.11$ & $16.1 \pm 0.39$ & 2.71 \\
NGC 4051 & $0.51 \pm 0.17$ & $0.93 \pm 0.01$ & $2.5 \pm 0.08$ & $3.77 \pm 0.07$ & 4.74 \\
MCG 6-30-15 & $5.32 \pm 0.12$ & $0.79 \pm 0.02$ & $1.23 \pm 0.13$ & $12.3 \pm 0.5$ & 0.95 \\
Mkn 841 & $2.15 \pm 0.06$ & $0.79 \pm 0.05$ & $0.33 \pm 0.10$ & $5.8 \pm 0.19$ & 1.17 \\
NGC 7469 & $5.00 \pm 0.09$ & $1.04 \pm 0.04$ & $0.45 \pm 0.07$ & $9.35 \pm 0.2$ & 1.1 \\
Akn 120 & $8.06 \pm 0.16$ & $1.17 \pm 0.03$ & $0.69 \pm 0.07$ & $9.70 \pm 0.20$ & 1.43 \\
NGC 5548 & $1.32 \pm 0.05$ & $0.76 \pm 0.02$ & $0.69 \pm 0.10$ & $5.50 \pm 0.15$ & 1.23 \\
Mkn 509 & $2.19 \pm 0.08$ & $1.14 \pm 0.05$ & $1.02 \pm 0.13$ & $20.6 \pm 0.7$ & 1.73 \\
NGC 3516 & $3.07 \pm 0.05$ & $0.77 \pm 0.01$ & $0.90 \pm 0.08$ & $20.1 \pm 0.04$ & 1.05 \\
MCG 2-58-22 & $2.44 \pm 0.08$ & $1.19 \pm 0.08$ & $0.53 \pm 0.09$ & $3.84 \pm 0.11$ & 1.27 \\
NGC 7213 & $1.75 \pm 0.04$ & $1.03 \pm 0.16$ & $0.16 \pm 0.07$ & $16.3 \pm 0.37$ & 1.85 \\
\hline
\end{tabular}

Note: (1) Neutral column density in units of $10^{20} \mathrm{~cm}^{-2}$ (2) Edge threshold energy (keV) (3) Absorption depth at threshold (4)

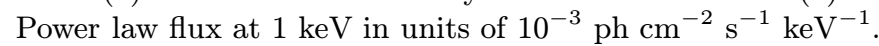

result is already clear from the Table: none of the models is a good description of the soft excess for all sources. In other words, the soft excess seems to be different in origin from source to source.

Let us now briefly discuss the results obtained for the different models.

\subsection{Black body}

As can be seen in Table 3 , the black body gives a satisfactory fit for 8 out of 13 sources (a ninth source, Fairall 9, is just above the "acceptability threshold" of $\chi_{\nu}^{2}=1.25$ ). The temperature is comprised between 50 and $140 \mathrm{eV}$. (Fig. 3, panel a); the sources identified with a cross are those for which the black body is the only acceptable model, those with an open triangle are the other sources for which the fit is good, while finally the solid circles identify sources which cannot be fitted with the black body). Parametrizing the relative intensity of the soft excess with the quantity $1-c_{\mathrm{pl}} / c_{\mathrm{tot}}$, where $c_{\mathrm{pl}}$ and $c_{\mathrm{tot}}$ are the power law and total count rate respectively, we found that such quantity is consistent with a constant, apart from NGC 4051 (Fig. 3, panel b).

A certain correlation between temperature and luminosity is apparent, expecially if NGC 4051, who has a much lower luminosity than the other sources, is excluded. It should be noted, however, that this correlation is largely due to three sources for which the black body is not the 
Table 5. Fit with reflection

\begin{tabular}{llllll}
\hline Obj. & $N_{\mathrm{H}}^{1}$ & $E_{0}^{2}$ & $C_{\text {refl }}^{3}$ & $C_{\mathrm{pl}}^{4}$ & $\chi_{\nu}^{2}$ \\
Mkn 335 & $3.17 \pm 0.06$ & $0.67 \pm 0.03$ & $12.7 \pm 1.7$ & $6.17 \pm 0.13$ & 1.52 \\
Fairall-9 & $2.07 \pm 0.07$ & $0.90 \pm 0.05$ & $12.2 \pm 0.7$ & $9.58 \pm 0.26$ & 1.36 \\
NGC 3783 & $11.3 \pm 0.33$ & $0.68 \pm 0.01$ & $47.0 \pm 3.8$ & $8.11 \pm 0.13$ & 5.09 \\
NGC 4051 & $0.94 \pm 0.06$ & $0.69 \pm 0.02$ & $9.72 \pm 0.62$ & $1.83 \pm 0.05$ & 1.48 \\
MCG 6-30-15 & $6.26 \pm 0.32$ & $0.60 \pm 0.03$ & $25.8 \pm 5.7$ & $8.52 \pm 0.18$ & 1.43 \\
Mkn 841 & $2.70 \pm 0.20$ & $0.49 \pm 0.04$ & $7.4 \pm 1.2$ & $5.80 \pm 0.50$ & 1.03 \\
NGC 7469 & $5.21 \pm 0.12$ & $0.83 \pm 0.05$ & $6.4 \pm 1.0$ & $7.81 \pm 0.14$ & 0.91 \\
Akn 120 & $8.43 \pm 0.21$ & $0.97 \pm 0.04$ & $9.11 \pm 0.45$ & $7.23 \pm 0.16$ & 0.99 \\
NGC 5548 & $1.78 \pm 0.16$ & $0.49 \pm 0.03$ & $8.1 \pm 2.0$ & $4.51 \pm 0.07$ & 1.31 \\
Mkn 509 & $2.53 \pm 0.14$ & $0.84 \pm 0.06$ & $28.8 \pm 4.2$ & $14.2 \pm 0.6$ & 1.31 \\
NGC 3516 & $3.59 \pm 0.15$ & $0.58 \pm 0.02$ & $29.2 \pm 5.1$ & $15.3 \pm 0.2$ & 1.93 \\
MCG 2-58-22 & $2.64 \pm 0.11$ & $0.90 \pm 0.07$ & $3.11 \pm 0.52$ & $3.10 \pm 0.10$ & 1.08 \\
NGC 7213 & $3.03 \pm 0.25$ & $0.45 \pm 0.03$ & $48.8 \pm 2.1$ & $15.9 \pm 0.4$ & 1.07 \\
\hline
\end{tabular}

Note: (1) Neutral column density in units of $10^{20} \mathrm{~cm}^{-2}$ (2) Cut off energy (keV) (3) Normalization of reflected component in units of $10^{-3} \mathrm{ph} \mathrm{cm}^{-2} \mathrm{~s}^{-1} \mathrm{keV}^{-1}$ (4) Power law flux at $1 \mathrm{keV}$ in units of $10^{-3} \mathrm{ph} \mathrm{cm}^{-2} \mathrm{~s}^{-1} \mathrm{keV}^{-1}$.

only model which provides a good fit. It is therefore possible that this behaviour is an artifact of attributing a temperature to a component which is completely different in origin, like the other two under investigation. In any case, these results argue against a pure standard disc origin for the soft excess, because in this case the temperature would decrease with luminosity (at least assuming a constant accretion rate per unit mass of the black hole).

Finally, it is interesting to note that also for the sources where the black body model is not statistically acceptable, the temperature and the relative normalization of the soft excess are similar to the average values.

An example of a source (Mrk 509) for which a black body model provides a good fit is presented in the upper panel of Fig. 4.

\subsection{Absorption edge}

Formally, the absorption edge gives a good fit for 5 objects. For 4 sources the edge energy is around $0.8 \mathrm{keV}$, corresponding to O VII and/or O VIII absorption. For NGC 7469 the edge would correspond to mildly ionized neon; such an edge is not expected to be present without a (strongest) oxygen edge, and we then consider the result physically meaningless. It is worth noticing that the 3 sources for which the absorption edge is the only successful model (MCG-6-30-15, NGC 5548 and NGC 3516, see Table 6) have all the "right" edge energy.

An example of a source (NGC 3516) for which an absorption edge fits well the data can be found in the middle panel of Fig. 4.

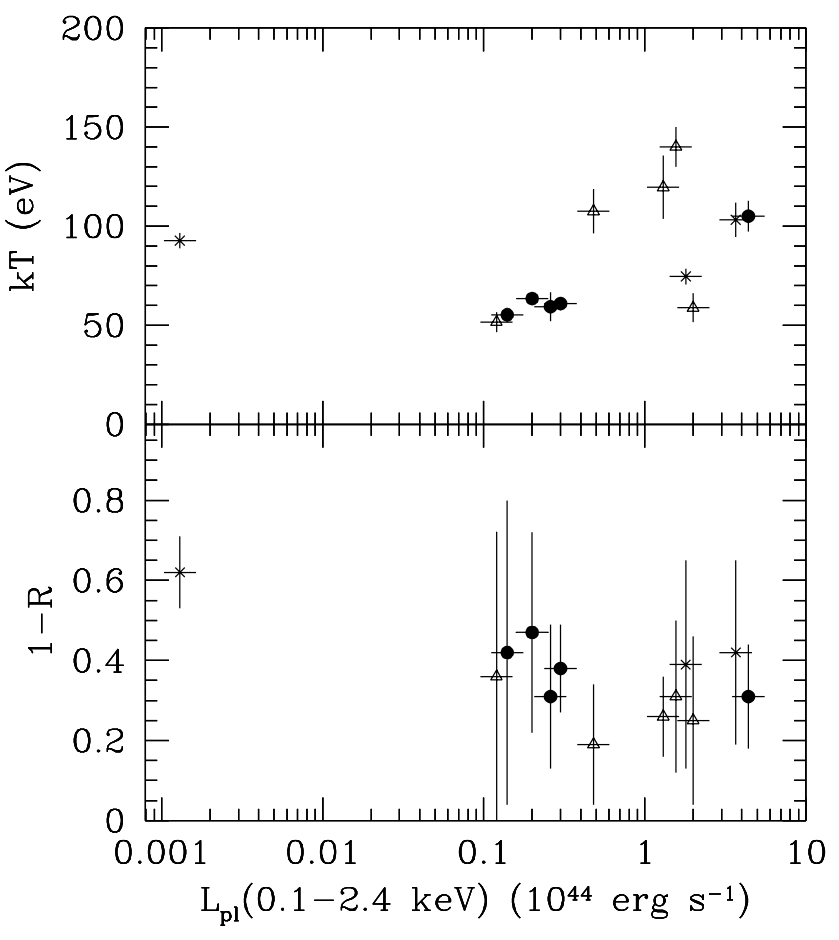

Fig. 3. Distribution of blackbody temperature (panel a) and intensity of the soft excess (panel b) vs. $L(0.1-2.4 \mathrm{keV})$. The objects for which the blackbody is the only model providing a good fit are identified with a cross; those for which is a good fit, but that are also well fitted by at least another model are identified by an open triangle; the other by a filled circle 


\subsection{Reflection}

This model gives a good fit for 5 sources but it is never the only good model. The edge energy is $\sim 0.45-0.5$, and then consistent with $\mathrm{C}$ VI, for two sources (Mkn 841 and NGC 7213); around $0.8-0.9$, and then consistent with O VIII, for other two sources (NGC 7469 and MCG-2-5822 ). For Ark 120 the fit is formally acceptable, but the edge energy (0.97) appears to be too high to be physically meaningful. Finally, in all the 4 sources in which the fit is good and $E_{0}$ meaningful, the normalizations of the reflection and primary components are of the same order, as expected for a $2 \pi$ illuminated matter (much different reflection components could occur only if the primary radiation were anisotropic, or as a result of a delayed response of the reflecting matter to changes in the primary emission).

In the lower panel of Fig. 4 an example of a source (NGC 7469) for which the reflection model provides a good fit is shown.

Table 6. Summary of model applicability

\begin{tabular}{lccc}
\hline Source & $b b$ & edge & refl. \\
Mkn 335 & $\times$ & & \\
Fairall-9 & & & \\
NGC 3783 & $\times$ & & \\
NGC 4051 & $\times$ & $\times$ & \\
MCG 6-30-15 & $\times$ & $\times$ & $\times$ \\
Mkn 841 & $\times$ & $\times$ & $\times$ \\
NGC 7469 & $\times$ & & $\times$ \\
Akn 120 & & $\times$ & \\
NGC 5548 & $\times$ & & \\
Mkn 509 & & $\times$ & \\
NGC 3516 & $\times$ & & $\times$ \\
MCG 2-58-22 & $\times$ & \\
NGC 7213 & $\times$ & & \\
\hline
\end{tabular}

Note: A cross indicates that the model in its simplest version gives a satisfactory fit.

\section{Notes on individual sources}

The complexity of the soft $\mathrm{X}$-ray emission is confirmed by the fact that, in a few sources, no model gives an acceptable fit. This is the case of NGC 3783 and Fairall 9 (even if for the latter the fit with the black body model is just above the acceptability threshold), while for another source (NGC 5548) the only acceptable model (the absorption edge), is just below the acceptability threshold. These three objects may thus represent the case when the spectral complexity of the soft X-ray excess shows clearly due to a combination of statistics and specific emission characteristics.

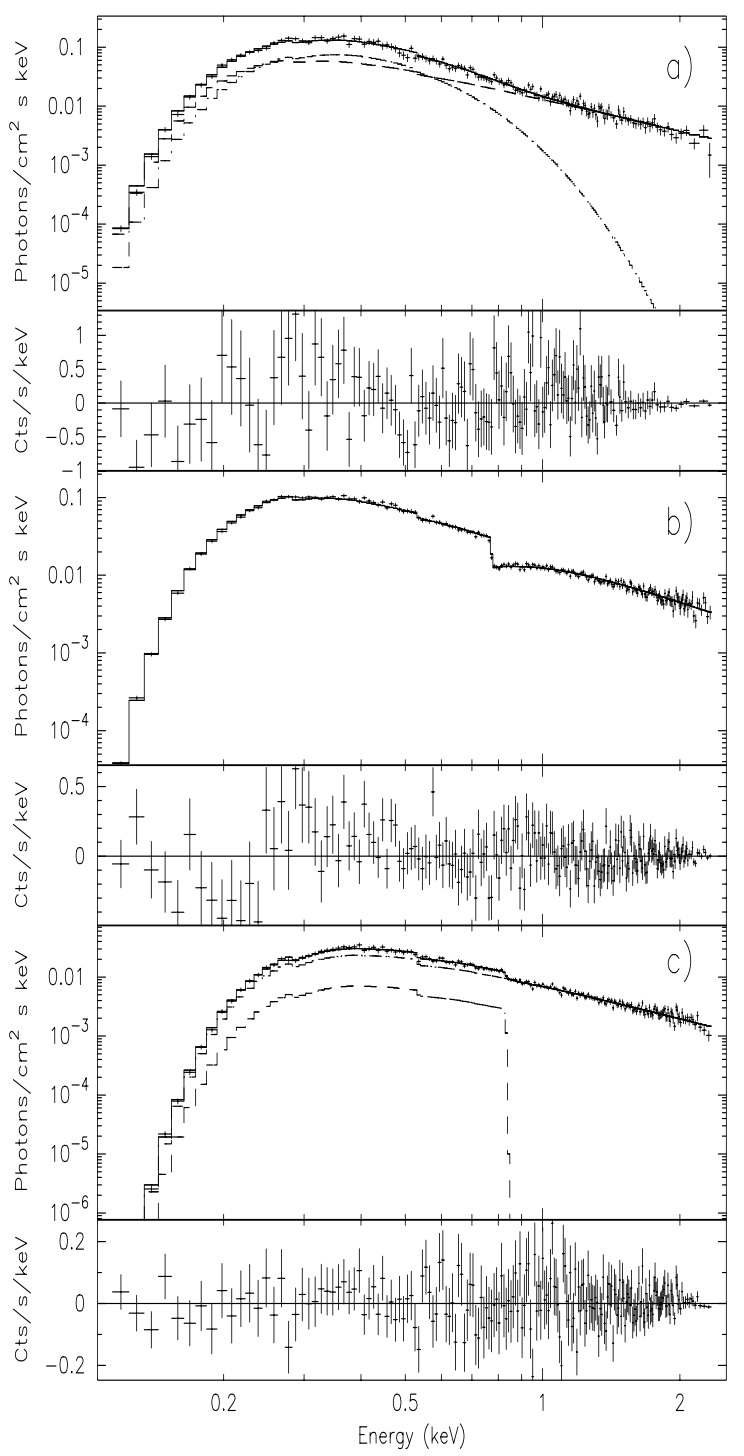

Fig. 4. Unfolded spectra and residuals for: a) black body fit to Mrk 509; b) absorption edge fit to NGC 3516; c) reflection model fit to NGC 7469

We have therefore carried out a more detailed analysis, being guided in the choice of more complex models by realistic scenarios. A first case is that of a warm absorber covering the central source where an accretion disk produces a soft excess either by thermal emission or by reflection. A second case is that of an emission line added to the two models in which it can be expected, i.e. the absorption edge and the reflection model. In fact, warm absorbers can manifest themselves not only through absorption features, but also with emission lines (e.g. Netzer 1993), provided that the covering factor is a significant fraction of $4 \pi$. Also in reflection models, strong line emission is predicted (Ross \& Fabian 1993). 
Due to the limited energy resolution of the PSPC, the number of free parameters in each fit cannot exceed $4-5$. When adding models, therefore, we fixed some fit parameters in order to never exceed 5 free parameters. In particular we fixed the absorption column density to the Galactic value.

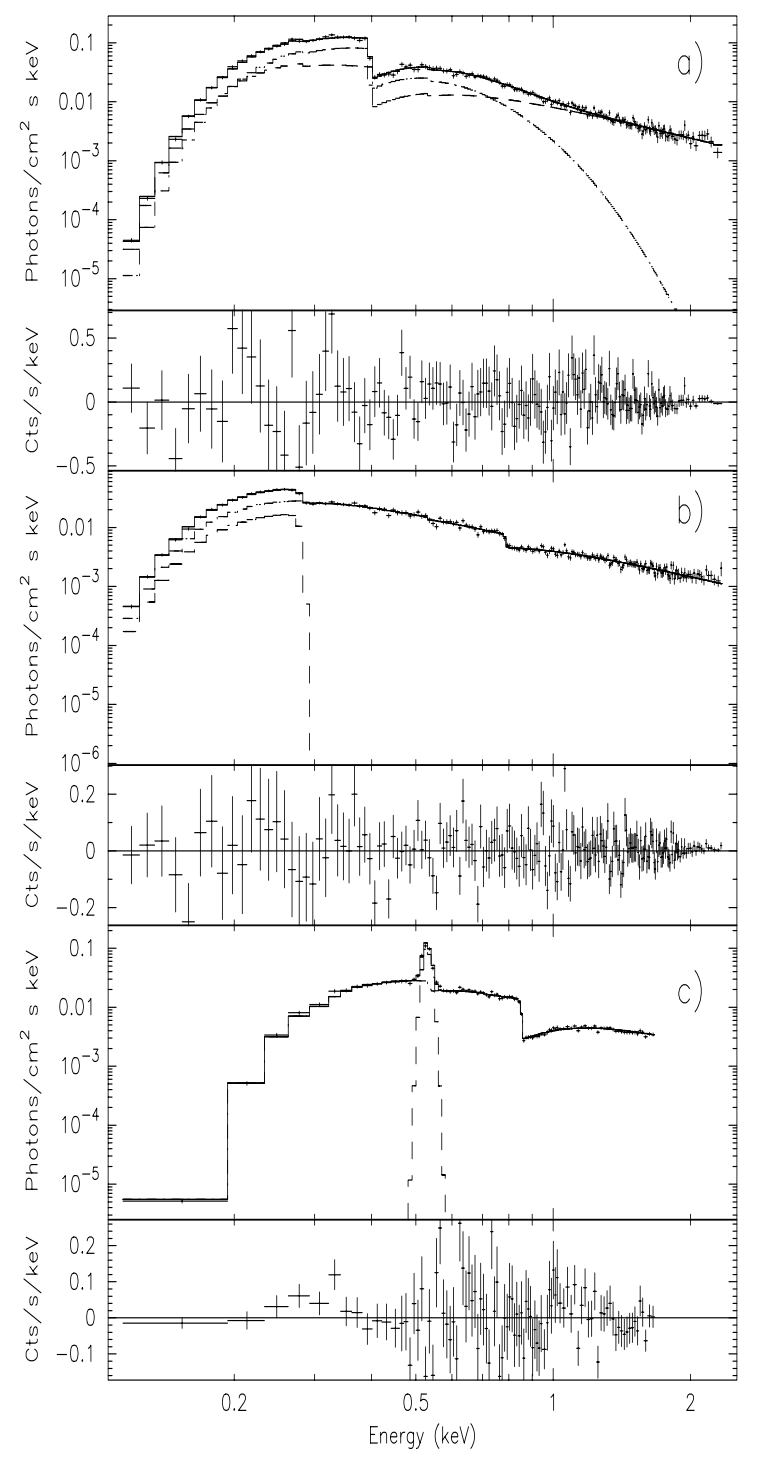

Fig. 5. Unfolded spectra and residuals for: a) absorption edge plus black body model for Fairall 9 ; b) edge plus reflection model for NGC 5548; c) edge plus line for NGC 3783

\subsection{Fairall 9}

A simultaneous Ginga-ROSAT observation of Fairall 9 suggested (despite the rather short exposure time, $578 \mathrm{~s}$, of the ROSAT observation, obtained during the All Sky
Survey) the presence of a soft excess (parametrized with a black body) and an emission line at $0.89( \pm 0.15) \mathrm{keV}$ whose identification is not obvious (Pounds et al. 1994).

The presence of a "true" soft X-ray emission is also suggested by our analysis, as the black body model gives the best fit, and indeed a $\chi_{\nu}^{2}$ which is very close to our acceptability threshold. We first tried to add an absorption edge to the black body and the reflection models. The results are summarized in Table 7 . Both fits are acceptable ( $\chi_{\nu}^{2}$ of 0.96 and 1.16 , respectively) and the parameters are reasonable (the edge energies correspond to $\mathrm{C} \mathrm{V}$ in the fit with the black body, and $\mathrm{C}$ VI in the fit with the reflecton model. In the last fit, $E_{0}$ is close to the $\mathrm{O}$ VIII edge). It must be noted, however, that the normalization of the reflection component is about three times that of the primary power law. The unfolded spectrum, as well as the residuals, for the absorption edge plus black body fit is shown in panel (a) of Fig. 5 .

We then tried to add an emission line (as suggested by Pounds et al. 1994) to the absorption edge or the reflection models. The fit with the absorption edge is unacceptable $\left(\chi_{\nu}^{2}=2.7\right)$, while that with the reflection model is acceptable $\left(\chi_{\nu}^{2}=1.1\right)$, with $E_{0}$ corresponding to the C VI edge (see Table 7), with a line energy of $0.76 \mathrm{keV}$ (the two closest lines are $\mathrm{O}$ VIII $\mathrm{K} \alpha$ recombinatin line at $0.65 \mathrm{keV}$ and the $\mathrm{O}$ VII recombination directly to the ground state at $\gtrsim 0.74 \mathrm{keV}$ ). Again, however, the normalization of the reflection component is about three times that of the primary power law.

We therefore conclude that in Fairall 9 a "true" emission is likely to be present, and that a further feature (probably an edge) is strongly suggested by the data.

\section{2. $N G C 5548$}

For NGC 5548 an acceptable fit is obtained with the absorption edge (and in fact the presence of an highly ionized oxygen edge is well established since Nandra et al. 1993, who analysed the same observation discussed here). However, the $\chi_{\nu}^{2}$ of 1.23 is close to the "acceptability threshold", suggesting that this model alone is possibly not sufficient to fully describe the PSPC spectrum. Indeed, both Nandra et al. (1993) and Done et al. (1995) suggest the presence of a "true" soft excess.

In fact, the inclusion of a black body or a reflection model in the absorption edge fits reduces significantly the $\chi_{\nu}^{2}$, which becomes about 1 in both cases. In the reflection model fit, $E_{0}$ is consistent with the $\mathrm{C}$ V edge, but the normalization of the reflection component is twice that of the primary radiation. The best fit parameters are summarized in Table 8 while the unfolded spectrum and the residuals for the edge plus reflection fit is shown in panel (b) of Fig. 5 .

We tried also to add an emission line to either the absorption edge or reflection model. In the former case, the fit is completely unacceptable $\left(\chi_{\nu}^{2}=1.62\right)$, while in the 
Table 7. Fits to Fairall 9

\begin{tabular}{ll}
\hline \multicolumn{2}{c}{ Absorption edge + black body $\left(\chi_{\nu}^{2}=0.96,184\right.$ d.o.f. $)$} \\
\hline$N_{\mathrm{H}}\left(10^{20} \mathrm{~cm}^{-2}\right)$ & 2.82 (fixed) \\
$C_{\mathrm{pl}}\left(10^{-3} \mathrm{ph} \mathrm{cm}^{-2} \mathrm{~s}^{-1}\right)$ & $9.47 \pm 0.28$ \\
$\Gamma \mathrm{pl}$ & 1.94 (fixed) \\
$E(\mathrm{keV})$ & $0.40_{-0.12}^{+0.04}$ \\
$\tau$ & $1.63_{-0.34}^{+2.97}$ \\
$k T(\mathrm{keV})$ & $0.107_{-0.004}^{+0.004}$ \\
$C_{\mathrm{bb}}\left(10^{-3} \mathrm{erg} \mathrm{s}^{-1} \mathrm{kpc}^{-2}\right)$ & $0.48 \pm 0.03$
\end{tabular}

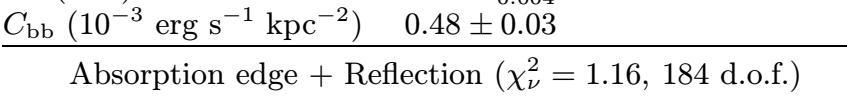

\begin{tabular}{ll}
$N_{\mathrm{H}}\left(10^{20} \mathrm{~cm}^{-2}\right)$ & 2.82 (fixed) \\
$C_{\mathrm{pl}}\left(10^{-3} \mathrm{ph} \mathrm{cm}^{-2} \mathrm{~s}^{-1}\right)$ & $10.2 \pm 0.2$ \\
$\Gamma \mathrm{pl}$ & 1.94 (fixed) \\
$E(\mathrm{keV})$ & $0.50_{-0.01}^{+0.02}$ \\
$\tau$ & $1.12_{-0.10}^{+0.11}$ \\
$E_{0}(\mathrm{keV})$ & $0.88_{-0.02}^{+0.04}$ \\
$C_{\mathrm{refl}}\left(10^{-3} \mathrm{ph} \mathrm{cm}^{-2} \mathrm{~s}^{-1}\right)$ & $31.5 \pm 1.2$ \\
\hline
\end{tabular}

Emission line + Reflection $\left(\chi_{\nu}^{2}=1.10,184\right.$ d.o.f. $)$

$\begin{array}{ll}N_{\mathrm{H}}\left(10^{20} \mathrm{~cm}^{-2}\right) & 2.82 \text { (fixed) } \\ C_{\mathrm{pl}}\left(10^{-3} \mathrm{ph} \mathrm{cm}^{-2} \mathrm{~s}^{-1}\right) & 9.78 \pm 0.22 \\ \Gamma \mathrm{pl} & 1.94 \text { (fixed) } \\ E_{\text {line }}(\mathrm{keV}) & 0.76_{-0.03}^{+0.02} \\ \sigma_{\text {line }}(\mathrm{keV}) & 0.01 \text { (fixed) } \\ I_{\text {line }}\left(10^{-3} \mathrm{ph} \mathrm{cm}^{-2} \mathrm{~s}^{-1}\right) & 2.13_{-0.28}^{+0.37} \\ E_{0}(\mathrm{keV}) & 0.50 \pm 0.02 \\ C_{\text {refl }}\left(10^{-3} \mathrm{ph} \mathrm{cm}^{-2} \mathrm{~s}^{-1}\right) & 32.8 \pm 1.1\end{array}$

latter case the fit is just above our acceptability threshold $\left(\chi_{\nu}^{2}=1.26\right)$ with a line energy consistent with the O VII recombination line, and $E_{0}$ consistent with $\mathrm{C}$ V (again, the normalization of the reflection component is twice that of the primary radiation). The best fit parameters for the latter case are also summarized in Table 8.

\section{3. $N G C 3783$}

NGC 3783 was by far the most intractable case in our previous analysis. All the three models are completely unacceptable, the best fit being given by the absorption edge with $\chi_{\nu}^{2}=2.7$. This cannot be simply due to the relative high statistic of the ROSAT observation, as there are sources with similar or even greater total number of counts which are well fitted by one or more models. Even excluding all channels above $2 \mathrm{keV}$ (see below) the fits remain unacceptable. Therefore, there must be something intrinsic in this source which prevents any simple model to fit satisfactorily.

The clue is given by an ASCA observation (George et al. 1995) which discovered in the spectrum of this source a line emission identified with the $\mathrm{O}$ VII $\mathrm{K} \alpha$
Table 8. Fits to NGC 5548

\begin{tabular}{ll}
\hline \multicolumn{2}{c}{ Absorption edge + black body $\left(\chi_{\nu}^{2}=1.01,194\right.$ d.o.f. $)$} \\
\hline$N_{\mathrm{H}}\left(10^{20} \mathrm{~cm}^{-2}\right)$ & 1.93 (fixed) \\
$C_{\mathrm{pl}}\left(10^{-3} \mathrm{ph} \mathrm{cm}^{-2} \mathrm{~s}^{-1}\right)$ & $5.27 \pm 0.17$ \\
$\Gamma \mathrm{pl}$ & 1.81 (fixed) \\
$E(\mathrm{keV})$ & $0.78 \pm 0.03$ \\
$\tau$ & $0.50_{-0.11}^{+0.12}$ \\
$k T(\mathrm{keV})$ & $0.046_{-0.006}^{+0.008}$ \\
$C_{\mathrm{bb}}\left(10^{-3} \mathrm{erg} \mathrm{s}^{-1} \mathrm{kpc}^{-2}\right)$ & $0.069_{-0.008}^{+0.015}$ \\
\hline \multicolumn{1}{c}{$\mathrm{Absorption} \mathrm{edge}+$ Reflection $\left(\chi_{\nu}^{2}=1.00,194\right.$ d.o.f. $)$} \\
\hline & $1.93($ fixed $)$ \\
$N_{\mathrm{H}}\left(10^{20} \mathrm{~cm}^{-2}\right)$ & $5.3 \pm 0.2$ \\
$C_{\mathrm{pl}}\left(10^{-3} \mathrm{ph} \mathrm{cm}^{-2} \mathrm{~s}^{-1}\right)$ & 1.81 (fixed) \\
$\Gamma \mathrm{pl}$ & $0.79_{-0.03}^{+0.02}$ \\
$E(\mathrm{keV})$ & $0.53_{-0.11}^{+0.10}$ \\
$\tau$ & $0.28_{-0.01}^{+0.12}$ \\
$E_{0}(\mathrm{keV})$ & $8.5 \pm 1.2$ \\
$C_{\mathrm{refl}}\left(10^{-3} \mathrm{ph} \mathrm{cm}^{-2} \mathrm{~s}^{-1}\right)$ & 1.2 \\
\hline \multicolumn{2}{c}{$\mathrm{Emission} \mathrm{line}+$ Reflection $\left(\chi_{\nu}^{2}=1.26,194\right.$ d.o.f. $)$} \\
\hline
\end{tabular}

recombination line at $0.57 \mathrm{keV}$. There is evidence, even if less compelling, also for the $\mathrm{O}$ VIII line at $0.65 \mathrm{keV}$. We then added a narrow emission line to both the absorption edge and reflection models.

At a first glance, the inclusion of the line does not make the fit acceptable if the whole energy band is used. However, the inspection of the residuals reveals a systematic excess above $\sim 2 \mathrm{keV}$, maybe due to calibration problems (this is the only source in which we have found such a problem). Excluding all channels above $2 \mathrm{keV}$, the reflection + line fit remains fully unacceptable $\left(\chi_{\nu}^{2}=2.7\right)$, but the absorption edge plus line fit turns out to be marginally acceptable $\left(\chi_{\nu}^{2}=1.23\right.$; see panel (c) of Fig. 5$)$. The best fit parameters are summarized in Table 9.

For the sake of completeness we tried also to add an absorption edge to the black body or the reflection models. In the latter case the fit is unacceptable $\left(\chi_{\nu}^{2}=\right.$ $1.64)$; on the contrary, in the former case it is acceptable $\left(\chi_{\nu}^{2}=1.15\right)$, with an edge energy close to that of O VII. The best fit parameters are summarized in Table 9. Even if the edge + black body model gives a better fit, the edge 
plus line fit seems to be preferred after the ASCA result mentioned above.

Table 9. Fits to NGC 3783

\begin{tabular}{ll}
\hline \multicolumn{2}{c}{ Absorption edge + black body $\left(\chi_{\nu}^{2}=1.15,94\right.$ d.o.f. $)$} \\
\hline$N_{\mathrm{H}}\left(10^{20} \mathrm{~cm}^{-2}\right)$ & 9.41 (fixed) \\
$C_{\mathrm{pl}}\left(10^{-3} \mathrm{ph} \mathrm{cm}^{-2} \mathrm{~s}^{-1}\right)$ & $11.2 \pm 0.06$ \\
$\Gamma \mathrm{pl}$ & 2.11 (fixed) \\
$E(\mathrm{keV})$ & $0.77_{-0.02}^{+0.011}$ \\
$\tau$ & $1.87_{-0.19}^{+0.20}$ \\
$k T(\mathrm{keV})$ & $0.137_{-0.011}^{+0.014}$ \\
$C_{\mathrm{bb}}\left(10^{-3} \mathrm{erg} \mathrm{s}^{-1} \mathrm{kpc}^{-2}\right)$ & $0.22 \pm 0.03$ \\
\hline \multicolumn{2}{c}{$\mathrm{Absorption} \mathrm{edge}+$ emission line $\left(\chi_{\nu}^{2}=1.23,94\right.$ d.o.f. $)$} \\
\hline$N_{\mathrm{H}}\left(10^{20} \mathrm{~cm}^{-2}\right)$ & $9.41($ fixed $)$ \\
$C_{\mathrm{pl}}\left(10^{-3} \mathrm{ph} \mathrm{cm}^{-2} \mathrm{~s}^{-1}\right)$ & $13.0_{-0.5}^{+0.6}$ \\
$\Gamma \mathrm{pl}$ & 2.11 (fixed) \\
$E(\mathrm{keV})$ & $0.85_{-0.01}^{+0.02}$ \\
$\tau$ & $1.56 \pm 0.14$ \\
$E_{\text {line }}(\mathrm{keV})$ & $0.53_{-0.02}^{+0.01}$ \\
$\sigma_{\text {line }}(\mathrm{keV})$ & 0.01 (fixed) \\
$I_{\text {line }}\left(10^{-3} \mathrm{ph} \mathrm{cm}^{-2} \mathrm{~s}^{-1}\right)$ & $5.5_{-0.6}^{+0.5}$ \\
\hline
\end{tabular}

\subsection{The case of $N G C 4051$}

In their analysis of the ASCA observations of this source, Guainazzi et al. (1997) attribute the observed spectral variations to changes in the slope of the intrinsic power law correlated with the luminosity of the object. The slope varies from $\Gamma=1.55$ to $\Gamma=2.0$ when $L(2-10 \mathrm{keV})$ goes from $3.510^{41} \mathrm{erg} \mathrm{s}^{-1}$ to $810^{41} \mathrm{erg} \mathrm{s}^{-1}$. Assuming this relationship, the intrinsic slope corresponding to the ROSAT luminosity would be $\Gamma \sim 1.4$. We then repeated the fits adopting for the intrinsic slope this very conservative value. The results are basically unchanged: the only acceptable model remains the blackbody $(\chi=1.02)$, with a relative normalization greater by about $30 \%$. The other parameters are similar to those obtained previously within $20 \%$.

\subsection{Other sources}

From the previous analysis on Fairall 9, NGC 5548 and NGC 3783 we have learned that complex scenarios may be acceptable when simple ones are not.

Since for all the remaining sources at least one model provides a good fit, we could not draw any significant information from a more detailed analysis. Of course any mixture of models will provide a good representation.

However, we had a further feedback from the analysis of the three sources that is important to verify.
Namely, in the case of warm absorber or reflection models, the presence of emission lines is possible. We have therefore added an emission line to all the sources where those two models were not able to fit the data. In most cases, the fit turns out to be formally acceptable (which is not surprising, as the number of free parameter is now equal to six, and the data are therefore somewhat overfitted), but the derived value of the line and/or edge energies are physically meaningless. In particular, this is the case for the edge model for Mrk 335, Ark 120, Mkn 509, MCG-2-58-22 and NGC 7213, while the same model for NGC 4051 remains statistically unacceptable. For the reflection model, the fit is unacceptable for MCG-6-30-15, while unphysical for Mkn 509. For Mrk 335, any line is set to zero. Finally, for NGC 4051 and NGC 3516 the fit is acceptable; the line energy is consistent with $\mathrm{C} \mathrm{V}$, while $E_{0}$ is consistent with $\mathrm{O}$ VII, a situation which seems rather unplausible.

We therefore conclude that, in general, the inability of the warm absorber or reflection models to fit the spectrum of many sources is not due to the inadequacy of the parameterization adopted.

\section{Discussion and conclusions}

We have studied the properties of the soft excesses for the Nandra \& Pounds (1994) sample of hard X-ray selected Seyfert 1 galaxies observed by Ginga. After excluding the objects with line-of-sight absorption in excess of $10^{21} \mathrm{~cm}^{-2}$ and/or with a total number of counts less than 2000, we obtained for 13 out of the 14 remaining sources a ROSAT power law index significantly steeper than the Ginga power law index, indicating the presence of a soft excess. We then fitted these 13 sources with three different models for the soft excess: a black body, an absorption edge and a reflection model (in all these fits the power law index of the hard component has been kept fixed to the Ginga value, implicitely assuming lack of spectral variability).

The main results can be summarized as follows:

- No model is able to fit all the sources in our sample.

- The black body model is the most successful, providing a good fit for 8 sources. The absorption edge gives formally a good fit for 5 sources, but for one of them (NGC 7469) the edge energy does not correspond to one of the expected transition. Exactly the same statistic occurs for the reflection model, Ark 120 being the source in which the characteristic energy is meaningless. The addition of an emission line to the edge or the reflection models when they are not able to fit well the data results, in most sources, in either a still unacceptable fit or in an acceptable fit but with physically meaningless parameters.

- For two sources, Fairall 9 and NGC 3783, no model gives acceptable fits (even if for Fairall 9 the black body is just beyond acceptability). For both sources, 
fits becomes acceptable after addition of either a black body component or an emission line.

- The only acceptable fit for NGC 5548 is that with the absorption edge, but the $\chi_{\nu}^{2}$ is very close to the boundary. The fit is significantly improved when a black body or a reflection model is added.

We therefore conclude that the soft excess phenomenon is complex and probably different from source to source. In some sources, more than one of the models discussed here are likely to occur simultaneously. This is not surprising, because the models are not mutually exclusive. Regarding the black body model, which is the most successful one, there is no strong evidence of correlation between temperature and luminosity. This could argue against simple accretion disc models, even if, after excluding NGC 4051, the range of luminosities spanned by our sample does not permit any definitive conclusion.

The analysis presented here is based on the assumption of absence of spectral variability. Even if this assumption appears realistic, as spectral variability has been observed up to now only in a handful of sources, it would obviously be desirable to determine simultaneously the shapes of the soft excess and the hard component. Besides, simultaneous observation would give informations on correlated variability, and therefore would further constrain models. Broad band observations, like those that will be performed by BeppoSAX, are expected to shed new light on the soft excess problem.

Acknowledgements. We thank G.C. Perola for a critical reading of the paper and suggestions and E. Massaro for useful discussions.

\section{References}

Allen C., 1973, Astrophysical quantities. Athlone Press

Arnaud K., et al., MNRAS 217, 105

Done C., Pounds K.A., Nandra K., Fabian A.C., 1995, MNRAS 275,1003

Fiore F., Perola G.C., Matsuoka M., Yamauchi M., Piro L., 1992, A\&A 262, 37

Fiore F., Matt G., Nicastro F., 1997, MNRAS 284, 731

George I.M., Turner T.J., Netzer H., 1995, ApJ 438, L67

Guainazzi M., Mihara T., Otani C., Matsuoka M., 1997, PASJ 48,1

Matt G., Fabian A.C., Ross R.R., 1993, MNRAS 264, 839

Nandra K., Pounds K.A., 1992, Nat 359, 215

Nandra K., et al., 1993, 260, 111

Nandra K., Pounds K.A., 1994, MNRAS 268, 405

Netzer H., 1993, ApJ 411, 594

Pfeffermann, et al., 1987, Proc. SPIE 733, 519

Perola G.C., et al., 1986, ApJ 306, 508

Piro L., et al., 1997, A\&A 319, 74

Pounds K.A., Nandra K., Fink H.H., Makino F., 1994, MNRAS 267, 193

Ross R.R., Fabian A.C., 1993, MNRAS 261, 74

Trümper J., 1983, Adv. Space Res. 2, 241

Turner T.J., Pounds K.A., 1989, MNRAS 240, 833

Yaqoob T., Warwick R.S., 1991, MNRAS 248, 773

Walter R., Fink H.H., 1993, A\&A 274, 105

Walter R., et al., 1994, A\&A 285, 119

Zimmermann H.U., Belloni T., Izzo C., Kahabka P., 1994, EXSAS Users' Guide, MPE report

Życki P.T., Krolik J.H., Zdziarski A.A., Kallman T.R., 1994, ApJ 437, 597 